\title{
Las culturas juveniles: un campo de estudio; breve agenda para la discusión*
}

\author{
Rossana Reguillo \\ Universidad de Guadalajara, Departamento de Estudios de la Comunicación Social \\ Instituto Tecnológico de Estudos Superiores do Ocidente, Departamento de Estudios Socioculturales
}

Soy anarquista, soy neonazi, soy un esquinjed y soy ecologista. Soy peronista, soy terrorista, capitalista y también soy pacifista/ Soy activista, sindicalista, soy agresivo y muy alternativo. Soy deportista, politeísta y también soy buen cristiano/ $Y$ en las tocadas la neta es el eslam pero en mi casa sí le meto al tropical... Me gusta tirar piedras, me gusta recogerlas, me gusta ir a pintar bardas y después ir a lavarlas.

Café Tacuba

Creo, por tanto, que la dimensión epistemológica de la reivindicación de la subjetividad es sólo un medio que nos acerca a la dimensión política. Jesús Ibáñez

Estas páginas intentan cuestionar los modos en que desde el campo cultural han sido pensadas las

* Reproduzido de G. M. Carrasco, Gabriel Medina (comp.). Aproximaciones a la diversidad juvenil. México: El Colegio de México, Centro de Estudios Sociológicos, 2000, com autorização da autora. culturas juveniles que, caracterizadas por sus sentidos múltiples y móviles, incorporan, desechan, mezclan, inventan símbolos y emblemas, en continuo movimiento que las vuelve difícilmente representables en su ambigüedad.

Para este cuestionamiento, el primer supuesto que se asume como punto de partida, es el de la enorme diversidad que cabe en la categoría "jóvenes": estudiantes, bandas, punks, milenaristas, empresarios, ravers, desempleados, sicarios, pero todos hijos de la modernidad, de la crisis y del desencanto.

Un segundo supuesto, entonces, lo constituye el contexto en tanto referente-mundo en el cual habitan estos nomádicos sujetos: el de un orden social marcado por la migración constante, el mundo globalizado, el reencuentro con los localismos, las tecnologías de comunicación, el desencanto político, el desgaste de los discursos dominantes y el deterioro de los emblemas aglutinadores, aunados a la profunda crisis estructural de la sociedad mexicana, como parte indisociable del escenario en el que cotidianamente miles de jóvenes semantizan el mundo y se lo apropian. 
Ello representa una enorme complejidad que vuelve imposible articular un solo campo de representaciones porque el sentido está siempre siendo, armándose en un continuum simbólico que desvanece fronteras, márgenes y límites.

De acuerdo con estos supuestos, la discusión que aquí se plantea está organizada en tres partes o ejes temáticos.

a) En una primera parte se analizan los discursos que han producido conocimiento sobre los jóvenes. A partir de una revisión de la literatura especializada disponible se buscó el conjunto de supuestos que han orientado, en el país, la mirada sobre los jóvenes, como insumo fundamental para arribar a una reflexión crítica sobre los conceptos, las categorías y los enfoques utilizados. Se trata de una primera aproximación a la naturaleza, límites y condiciones del discurso que se ha producido sobre las culturas juveniles.

b) En un segundo momento se discute acerca de los "nuevos" escenarios tanto en lo que respecta al pensamiento sobre las culturas juveniles, como en lo que toca a sus territorios - materiales y simbólicos.

c) En el tercer momento se abordan las perspectivas y desafíos que para la investigación en ciencias sociales representa el campo de estudio de las culturas juveniles.

Es importante plantear de entrada que los jóvenes no representan una categoría unívoca. La juventud es una categoría construida culturalmente, no se trata de una "esencia" y, en tal sentido, la mutabilidad de los criterios que fijan los límites y los comportamientos de lo juvenil, está necesariamente vinculada a los contextos sociohistóricos, producto de las relaciones de fuerza en una determinada sociedad. Así, lo que estas páginas intentan es objectivar los modos en que los jóvenes son construidos por los estudiosos del tema, a partir de unos recortes y ejes particulares; y simultáneamente proponer algunos elementos de reflexión sobre un tema que, pienso. será clave en el transcurso de los próximos años, de manera especial para México y América Latina.

\section{Pensar a los jóvenes: la construcción cultural de la categoría}

Definir al joven en términos socioculturales implica, en primer lugar, no conformarse con las delimitaciones biológicas, como la de la edad. Se ha dicho que "la juventud no es más que una palabra" (Bourdieu, 1990) y hoy sabemos que las distintas sociedades en diferentes etapas históricas han planteado las segmentaciones sociales por grupos de edad de muy distintas maneras y que, incluso, para algunas sociedades este tipo de recorte no ha existido.

No se trata en estas páginas de rastrear las formas en que las distintas sociedades han construido la categoría "jóvenes"1 sino de destacar el error que representa pensar a este grupo social como un continuo temporal y ahistórico.

Para los efectos de este ensayo se señala que la juventud, como hoy la conocemos, es propiamente una invención de la posguerra que hizo posible el surgimiento de un nuevo orden internacional que conformó una geografía política en la que los vencedores accedían a inéditos estándares de vida e imponían sus estilos y valores.

Cobraba forma un discurso jurídico, un discurso escolar y una floreciente industria, que reivindicaban la existencia de los niños y los jóvenes como sujetos de derecho y, especialmente, en cuanto a los jóvenes, como sujetos de consumo.

Las sociedades del "primer mundo" alcanzaban una insospechada esperanza de vida, lo que tuvo repercusiones directas en la llamada vida socialmen-

${ }^{1}$ Para este fin, véase, por ejemplo, G. Levi y J C Schmitt (directores), História de los jóvenes. Taurus, 1996. También el excelente trabajo de recuperación histórica de Carles Feixa, $L a$ tribu juvenil, una aproximación transcultural a la juventud. Edizione L'Occhiello, Turín, 1988. 
te productiva y, por ende, la inserción de las generaciones de relevo tendía a posponerse. Los jóvenes debían ser retenidos durante un periodo más largo en las instituciones educativas. ${ }^{2} \mathrm{Al}$ mismo tiempo, emergía una poderosa industria cultural que ofrecía por primera vez bienes "exclusivos" para el consumo de los jóvenes.

En esta en emergencia de la juventud como sujeto social ha desempeñado un papel fundamental el paso de la ciudadanía civil a la ciudadanía política (Marshall, 1965), en el sentido de la complementación de los derechos individuales, la libertad, la justicia y la propiedad, con los derechos a participar en el espacio público.

Por tanto, puede considerarse que la realización tecnológica y sus repercusiones en la organización productiva y simbólica de la sociedad, la oferta y el consumo cultural y el discurso jurídico, se constituyen entonces en tres elementos que le dan sentido y especificidad al mundo juvenil, más allá de la fijación de unos límites biológicos de edad.

Sin embargo, se han insinuado ya algunas "líneas de fuga" que obligan a replantear la definición del sujeto juvenil; (re)definición que conecta directamente con lo que se ha llamado "ciudadanía cultural" (Rosaldo, 1990).

Primero, resulta evidente que la realización tecnologica y los valores que se le asocian, lejos de achicar la brecha entre los que tienen y los que no, entre los poderosos y los débiles, entre los que están dentro y los que están fuera, la ha incrementado. La

${ }^{2}$ En cuanto a control social sobre los grupos más jóvenes se encuentran, por ejemplo, datos que señalan que en la Europa judía de 1660, la instrucción llegaba hasta los 13 años en el caso de los varones pudientes y a los 10 años en caso de los varones pobres, que debían entrar a servir a esta edad. Puede notarse cómo a medida que pasa el tiempo va aumentando la ampliación de los rangos de edad para la instrucción, que no es solamente una forma de distribución del conocimiento social sino además un mecanismo de control social. Véase Elliot Horowitz, Los mundos de la juventud judía en Europa:1300-1800, en Levi y Schmitt, op. cit. posibilidad de acceso a una calidad de vida digna es hoy para 200 millones de latinoamericanos ${ }^{3}$ un espejismo. Si este dato se cruza con el perfil demográfico del continente mayoritariamente juvenil, no se requieren grandes planteamientos para inferir que uno de los sectores más vulnerables por el empobrecimiento estructural, es precisamente el de los jóvenes.

Segundo, en lo que toca a la adquisición de la ciudadanía, uno de cuyos soportes fundamentales es el derecho a la integración plena en la sociedad, el problema es complejo ya que el papel que la ciudadanía ha desempeñado en torno a la constitución y su vinculación con ciertas categorías sociales, es ambiguo y contradictorio. En México la ciudadanía se otorga a una edad en la que los jóvenes están muy lejos aún (dependiendo de los niveles socioeconómicos) de acceder a una plena integración al sistema productivo, tanto por el deterioro de los mecanismos de integración (crisis político-cultural), como por la incapacidad real de las instituciones para absorberlos (crisis político-económica).

Esto ha resultado en un discurso esquizofrénico, en el que se exige de los jóvenes, cuando hacen su entrada en el universo de los derechos y deberes ciudadanos, ciertos comportamientos sociales, culturales y políticos, pero no hay alternativas reales de inserción económica. Puede señalarse aquí, a manera de ejemplo, el debate en torno a la disminución de la edad penal, de cuyas múltiples repercusiones se señala exclusivamente la contradicción y el conflicto societal que implica fijar unos criterios "móviles" que otorgan parcialmente a una edad, penalizan a otra y no incorporan a los sujetos en un sentido pleno.

En tercer lugar, la importancia creciente de las industrias culturales en la construcción y reconfiguraciones constantes del sujeto juvenil es un hecho que sale al paso de cualquier observador. El vestua-

${ }^{3}$ América Latina comenzó la década de 1970 con 200 millones de pobres, es decir, con 70 millones más de los que tenía en 1970, principalmente como resultado de la pobreza urbana (Roux, 1994). 
rio, la música y ciertos objetos emblemáticos constituyen hoy una de las más importantes mediaciones para la construcción identitaria de los jóvenes, elementos que se ofrecen no solo como marcas visibles de ciertas adscripciones sino fundamentalmente como lo que los publicistas llamam con gran sentido "un concepto, un estilo". Un modo de entender el mundo y un mundo para cada necesidad, en la tensión-identificación-diferenciación. Efecto simbólico - no por ello menos real - de identificarse con los iguales y diferenciarse de los otros, especialmente del mundo adulto.

Inexorablemente el mundo se achica y la juventud internacionalizada que se contempla a sí misma como espectáculo de los grandes medios de comunicación encuentra paradójicamente cn la homogeneización la posibilidad de diferenciarse y, sobre todo, la posibilidad de acceso a una ciudadanía cultural que no se detine mediante actos jurídicos, sino que se experimenta como el derecho a la igualdad en la afinación de la diferencia.

En estos territorios, en los de la cultura así experimentada, la juventud es un "estado", no una etapa de transición, ni un proceso de metamorfosis. De ahí el choque principal, en términos culturales, entre los diferentes discursos sociales en torno a los jóvenes.

Con excepciones, el Estado, la familia y la escuela siguen pensando a la juventud como una categoría de tránsito entre un estado y otro, como una etapa de preparación para lo que sí vale la juventud como futuro. Mientras que, para los jóvenes, su ser y su hacer en el mundo está anclado en el presente, lo que ha sido finamente captado por el mercado.

La construcción cultural de la categoría "joven", al igual que otras "calificaciones" sociales (mujeres e indígenas, entre otros) se encuentra en fase aguda de recomposición, lo que de ninguna manera significa que se piense, como ya se ha señalado, que había permanecido hasta hoy inmutable. Lo que resulta indudable es que los cambios planetarios han acelerado los procesos y han provocado crisis en los sistemas para pensar y nombrar el mundo. La juventud no es más que una palabra, una categoría construida, pero las categorías son productivas, hacen cosas, son simultáneamente productos del acuerdo social y productoras del mundo.

\section{Literatura sobre juventud: conceptos y categorías}

Partiendo del reconocimiento del carácter dinámico y discontinuo de los jóvenes, que no comparten en absoluto los modos de inserción en la estructura social, se plantea que sus esquemas de representación configuran campos de acción diferenciados y desiguales.

Pese a ello, en términos generales, la gran mayoría de los estudios sobre culturas juveniles no ha matizado suficientemente esta diferenciación, y la mayor parte de las veces ésta es abordada (y reducida) en función del tipo de "inserción" de los jóvenes en la sociedad.

En un primer acercamiento exploratorio y en términos de su vinculación con la estructura o sistema, en la literatura pueden reconocerse básicamente dos tipos de actores juveniles:

a) Los que pueden conceptualizarse como "incorporados" y que han sido analizados a través o desde su pertenencia al ámbito escolar o religioso; o bien, desde el consumo cultural.

b) Los "alternativos" o "disidentes" cuyas prácticas culturales han producido abundantes páginas y que han sido analizados desde su no-incorporación a los esquemas de la cultura dominante.

Desde luego este recorte es un tanto arbitrario (¿qué recorte analítico no lo es?) y sumamente grueso para los fines del análisis, pese a ello, resulta útil como una primera entrada que permita ir desentrañando cómo han sido pensados los jóvenes.

Si se acepta este primer recorte, el balance de los estudios se inclina tanto en términos cuantitativos como en lo referente a la relativa consolidación de lo que podría considerarse una "perspectiva" de estudio, del lado de los "alternativos" o "disidentes". En tan- 
to, sobre "los incorporados" la producción tiende a ser dispersa y escasa. ${ }^{4}$

Estas tendencias señalan que el interés de los estudiosos se ha centrado de manera prioritaria en aquellas formas de agregación, adscripción y organización juveniles que transcurren por fuera de las vías institucionales. Esta “selección” apunta a una cuestión que resulta vital y no es de ninguna manera "inocente" o "neutra": la pregunta por el sujeto.

Esta pregunta ha estado orientada por una intelección que, con sus matices y diferencias, reconoce las características y especificidades del sujeto juvenil. La casi imposibilidad de establecer márgenes fijos, "naturales al sujeto de estudio, ha obligado a una buena parte de los estudiosos de esta vertiente a situarse en los territorios de los propios jóvenes, lo que da como resultado una abundante cantidad de reportes, monografías, tesis, videos, que miran al joven como esencialmente contestario o marginal. ${ }^{5}$

Hay en estos estudios una tendencia fuerte a (con)fundir el escenario situacional con las representaciones profundas de estos jóvenes o, lo que es peor, a establecer una relación mecánica y transparente entre prácticas sociales y universos simbólicos.

Por ejemplo, la calle en tanto escenario "natural" asume en muchos de los estudios un papel de antagonista en relación con los espacios escolares o familiares y pocas veces ha sido pensada como espacio de extensión de los ámbitos institucionales en las prácticas juveniles. Los jóvenes en la calle parecerían

${ }^{4}$ Para obtener un panorama bastante completo véanse, por ejemplo, los dos tomos producto de la Reunión Nacional de Investigadores de la Juventud, celebrada en Querétaro a finales de 1996. En estos tomos se presenta una serie de "estados del arte" que recogen diez años de trabajos a proposito de la investigacióm sobre juventud en diferentes áreas temáticas (Pérez Islas y Maldonad, 1996).

5 "Marginal" se utiliza aquí en un sentido metafórico, para hacer alusión a una forma de respuesta "activa" al choque de valores. Para conocer una exposición más amplia véase Giddens (1995) y Maffesoli (1990). no tener vínculos con ningún tipo de institución, ajenos a cualquier normatividad y censura por parte del mundo adulto y oficial; de otro lado, prácticas como el lenguaje, los rituales de consumo cultural, las marcas de vestuario, al presentarse como diferentes y, en muchos casos, como atentadoras del orden establecido, han llevado a plantearlas como "evidencias" incuestionables del contenido liberador a priori de las culturas juveniles, sin ponerlas en contexto (deshistorizadas) o sin problematizarlas con la mediación de instrumentos de análisis que posibiliten trascender la dimensión descriptiva de los estudios. ${ }^{6}$

Esto nos lleva a un segundo planteamiento. A partir del interesante y acucioso análisis del estado de la cuestión sobre organización juvenil, realizado por Maritza Urteaga, ${ }^{7}$ se plantea aquí que en relación con los estudios sobre juventud, hechos en México en términos generales - pueden reconocerse dos momentos o tipos de conocimiento.

Un primer momento que para efectos prácticos ${ }^{8}$ puede ubicarse en la primera mitad de la década de los ochenta, estaría caracterizado tanto por acercamientos de tipo emic ${ }^{9}$ (específico, finalista, punto de vista interior), como por acercamientos de tipo etic (genérico, predictivo y exterior). Pero ambos tipos unidos por un tratamiento de carácter descriptivo.

Mientras que en el primer tipo es el punto de vista del "nativo" lo que prevalece, se asume por ende

${ }^{6}$ Por ejemplo, las innumerables posibilidades que ofrece el análisis discursivo: enunciación, semiótica, análisis pragmático, actos de habla etc.

${ }^{7}$ Véase Maritza Urteaga-Pozo, Organización juvenil, en José Antonio Pérez lslas y Elsa Patrícia Maldonado (coords.), Jóvenes : una evaluación del conocimiento; La investigación sobre la juventud en México 1986-1996. Causa Joven, México, 1996.

${ }^{8}$ Tomando como base las fechas de publicación de los estudios.

${ }^{9}$ Según la propuesta de Pike para el estudio de la conducta (retomada a su vez de Sapir) en la que se distinguen: "phonetics", que se ocupa de los sonidos en el sentido físico, y "phonemics", que trata los fonemas en sentido lingüístico (Pike, 1954). 
que todo lo "construido" y dicho al interior del sistema es necesariamente "la verdad"; mientras que en la segunda vertiente, lo que organiza el conocimiento proviene de las imputaciones de un observador externo al sistema, que no sabe (no puede, no quiere) dialogar con los elementos emic, es decir con las representaciones interiores o nativas.

Como se señaló antes, pese a las diferencias en la posición del observador, estos acercamientos comparten un enfoque descriptivo en el que no se explicitan las categorias y conceptos que orientan la mirada. Ello vuelve prácticamente imposible un diálogo epistémico entre perspectivas, convirtiendo las diferencias de apreciación en un forcejeo o tironeo estéril entre posiciones. Es decir, donde unos ven "anomia" y "desviaciones", otros ven "cohesión" y "propuestas". Los primeros tienden a recurrir al lenguaje normativo de la ciencia, a partir del cual "descalifican" el conocimiento "militante" producido por los segundos; mientras que estos últimos recurren a su posición interna - de intelectual orgánico - para descalificar las proposiciones de los primeros. ${ }^{10}$

Sin embargo, en la medida en que en términos generales ninguno de estos discursos trasciende lo descriptivo, el intercamhio posible queda atrapado en el nivel de la anécdota, de la interpretación "interesada" (en uno y otro caso), lo que desafortunadamente desemboca en una sustancialización de sujetos y prácticas.

Sin pretender aquí descalificar la cantidad de estudios producidos en esta época y sus aportes al

${ }^{10}$ Una ejemplificación de esto puede encontrarse en el trabajo de Gómez Jara que, a partir de un acercamiento de carácter psicosocial, proporcionó los primeros marcos conceptuales para analisar los comportamientos juveniles con énfasis en la violencia y la delincuencia (Gómez Jara, 1987, especialmente capítulo III). Para una ejemplificación del conocimiento producido de tipo "militante" pueden verse los primeros trabajos de Pablo Gaytán, entre otros, Notas sobre el movimiento juvenil. México: institucionalidad y marginalidad, Revista A, n 16, UAM-A(Universidad Autónoma Metropolitana Azcapatzalco), México, 1985. conocimiento de las culturas juveniles, es necesario apuntar que en términos generales, la producción de este periodo se caracteriza por una autocomplacencia que no asume de manera intencionada la construcción de un andamiaje teórico-metodológico que soporte los estudios realizados y que, en cambio, tiende a fijar una posición en torno al sujeto de estudio.

Hacia finales de la década de los años ochenta y principio de los noventa, puede reconocerse la emergencia de un nuevo tipo de discurso comprensivo en torno a los jóvenes. De carácter constructivista, relacional, que intenta problematizar no sólo al sujeto empírico de sus estudios, sino también a las "herramientas" que utiliza para conocerlo. Puede plantearse que se trata de perspectivas interpretativohermenéuticas, que intentan conciliar la oposición exterior-interior como parte de una tensión indisociable a la producción de conocimiento científico.

Vale la pena detenerse un momento para intentar ubicar aquí, en términos muy generales, el debate que en ciencias sociales ha influido de maneras diversas algunos de los estudios sobre juventud de este periodo.

Por ejemplo, el trabajo desarrollado por el sociólogo francés Pierre Bourdieu, que ha hecho énfasis en que su concepto de habitus ${ }^{11}$ es su intento (su apuesta) por superar la dicotomía planteada por la sociología clásica entre instituciones y sujetos, entre estructuras y prácticas, entre formas de control y formas de participación, o planteado en los propios términos de Bourdieu, entre el momento objetivo y el momento subjetivo de la cultura.

El británico Giddens, con su compleja y potente propuesta de "estructuración" social, que supone el papel activo de los sujetos en la constitución del mundo social, lo que metodológicamente implica trabajar en lo que él ha denominado una "doble hermenéutica",

${ }^{11}$ Muchas veces utilizado de manera abusiva, desprovisto de su tarea básica (servir como mediación teórico-metodológica entre las estructuras y las prácticas) y usado como equivalente aproblemático de "identidad", en el mejor de los casos, ya que también suele utilizarse en remplazo de "biografía". 
que a su vez está anclada en el viejo supuesto weberiano de la interpretación que hacen los actores de sus propias acciones. Para Giddens, el analista trabaja sobre estas interpretaciones convirtiéndose así su discurso en una interpretación de las interpretaciones.

Habermas coloca al centro de su teoría una subjetividad que se expresa por medio del lenguaje, para lo cual recupera y reformula como una categoría clave el concepto de "mundo de la vida" desarrollado por Husserl y la fenomenología. ${ }^{12}$ Metodológicamente ello significa reconocer al sujeto como la capacidad de referirse en actitud objetivante a las entidades del mundo y la capacidad de adueñarse de los objetos, sea teórica o prácticamente.

Estas formulaciones teóricas, pese a sus diferencias, se encuentran en el reconocimiento del papel activo de los sujetos sociales, de su capacidad de negociación con las instituciones, estructuras o sistemas (de acuerdo con la terminología propia de cada autor). Y, fundamentalmente, comparten la preocupación por el principio de "reflexividad", es decir, "pensar el pensamiento", en términos de lbáñez (1994), o la distancia entre un pensamiento que "toma" el mundo social y lo registra como datum, o sea, como dato empírico independiente del acto de conocimiento y de la ciencia que lo propicia (Bourdieu \& Wacquant, 1995), y un pensamiento que es capaz de hacer la crítica de sus propios procedimientos.

Esta pequeña desviación de la ruta principal es útil en tanto que permite ubicar comprensivamente la emergencia de un nuevo tipo de estudios y señalar los cambios habidos respecto al periodo anterior. Ello, desde luego, no significa que en la literatura revisada aparezcan de manera "explícita" estas posiciones, mucho menos estos autores. Pero si es posible reconocer una tendencia creciente a darle a los estudios sobre juventud un marco comprensivo-interpretativo que está anclado en tres dimensiones: la capacidad activa de los sujetos, el lenguaje no sólo como vehículo

${ }^{12}$ Para una discusión más amplia sobre este concepto, véase Reguillo (1996). sino como constructor de realidades, y la problematización constante de los propios supuestos de el(la) investigador(a).

De las perspectivas teórico-metodológicas aqui recuperadas, cabe hacer énfasis en que la vertiente de estudios interpretativos sobre las culturas juveniles ha incorporado, de maneras diversas, el reconocimiento del papel activo de los jóvenes, de su capacidad de negociación con sistemas e instituciones y de su ambigüedad en los modos de relación con los esquemas dominantes. Esto ha posibilitado, en términos generales, trascender las posiciones esencialistas: "o todo pérdida, o todo afirmación"; y encontrar otro nivel para la discusión que no se agota en la anécdota o en el dato empírico.

Aquí se asume que "las clasificaciones explícitas (edades de vida, época de la mayoría de edad etc.) evidentemente no poseen sino un valor indicativo. No bastan para definir los contextos de una historia social y cultural de la juventud" (Levi \& Schmitt, 1995).

En tal sentido, el segundo periodo o vertiente de estudios en el caso de México, puede considerarse abierto a partir de lo que podrían entenderse como los primeros trabajos claramente dirigidos en la línea de una "historia cultural" de la juventud ${ }^{13}$ y los que podrían ubicarse como los primeros trabajos que desde una perspectiva interdisciplinaria problematizan el discurso del sujeto juvenil (Reguilio, 1993; UrteagaPozo, 1993; Castillo, Zermeño \& Ziccardi, 1995).

Es decir, pueden considerarse, por un lado, la tarea de historizar sujetos y prácticas juveniles a la luz de los cambios culturales, rastreando orígenes, mutaciones, contextos político-sociales; y por otro lado, la perspectiva hermenéutica que rastrea la configuración de sentidos sociales, trascendiendo la descripción a través de las operaciones de construcción del objeto de estudio y con la mediación

${ }^{13}$ Por ejemplo el trabajo pionero de José Manuel Valenzuela, en publicaciones diversas y dos de sus libros, ;A la brava ése! El Colegio de la Frontera Norte, México, 1988 y Vida de barro duro, Colef/UdeG, Guadalajara, 1997. 
de herramientas analíticas. Si se está de acuerdo con Wallerstein en que los tres temas que se han conjuntado en los estudios culturales son:

[...] primero la importancia central, para el estudio de los sistemas sociales históricos, de los estudios de género y todos los tipos de estudios "no eurocéntricos"; segundo, la importancia del análisis histórico local, muy ubicado, que muchos asocian con una nueva actitud "hermenéutica"; tercero, la estimación de los valores asociados con las realizaciones tecnológicas y su relación con otros valores...

(Wallerstein, 1996, p. 71)

Puede argumentarse entonces que lo destacado en el periodo que va de finales de los ochenta a la década de los noventa en el estudio de las culturas juveniles no es ajeno a la perspectiva de los estudios culturales. ${ }^{14}$

En esta emergencia - de un modo constructivista y centralmente cultural - es importante señalar la importancia que ha tenido otra vertiente de trabajos que, abrevando en una larga tradición latinoamericana, se ubican en la perspectiva de crónica-periodística.

Se retomam aquí tres ejemplos clave, guardando las diferencias. En el caso de México, el trabajo de Carlos Monsiváis (1988), que ha sabido penetrar - y rescatar - con agudeza los elementos significativos y pertinentes para la comprensión de las formas culturales de la juventud.

Alonso Salazar (1998) en Colombia, que a partir de su incursión en los mundos del narcotráfico, del sicariato y de las comunas de Medellín, ha puesto al descubierto una situación descarnada y terriblemente compleja del mundo juvenil. Salazar ha sabido colocar simultáneamente la mirada del observador externo y la mirada del "nativo".

En el caso de Venezuela, puede señalarse el trabajo de Jose Roberto Duque y Boris Muñoz (1995), que han logrado incorporar, con gran sentido crítico,

${ }^{14}$ Pese a que tenga "cuentas pendientes", de lo que nos ocuparemos más adelante. las diferentes voces comprendidas en la problemática juvenil de Caracas. Hablan los jóvenes desde su precaria situación social, pero se incorporan también las voces de autoridades gubernamentales, representantes de la Iglesia, promotores sociales y analistas.

Por supuesto, estos autores no agotan el espectro de producciones que desde la crónica o el ensayo periodístico han posibilitado una mirada cualitativamente diferente sobre las culturas juveniles "alternativas" o "disidentes". Pero son suficientes para señalar los modos en que las ciencias sociales se han abierto a otro tipo de discursos.

El proyecto comprensivo respecto a las culturas juveniles requiere un segundo acercamiento para discutir los temas y los elementos que han sido problematizados. Empero, por cuestiones de carácter expositivo, primero nos ocuparemos de los discursos producidos en torno a los que aquí se han caracterizado como "los incorporados".

Se señaló ya que la literatura producida en torno a los jóvenes que transitan por las rutas "predecibles" tiende a ser dispersa y escasa. Otra característica muy importante de esta literatura es que en varios casos el objeto principal de estudio no lo constituyen los jóvenes, sino que son enfoques centrados, por ejemplo, en el aparato escolar, en las comunidades eclesiales de base, en las maquiladoras, en los sindicatos, cuyos autores están más interesados en los modos de funcionamiento de instituiciones y espacios que en las culturas juveniles. Los jóvenes aparecen entonces en su papel de "estudiantes", de "empleados", de "creyentes", de "obreros", y su especificidad como sujetos juveniles (más allá de las clasificaciones de edad) tiende a diluirse. ${ }^{15}$

${ }^{15}$ Este argumento se sostene a partir de la revisión de una gran cantidad de estudios empíricos producidos por la sociología del trabajo o por los investigadores de la educación y los valores. A manera de ejemplo de esta "disolución" del sujeto juvenil, véase Rafael Izquierdo (1996). Véase también Enrique Luengo (1996). Aunque se trata de estudios excelentes, tienden a perder las especificidades del sujeto. 
Es más bien el discurso cinematografico y literario el que ha logrado interesantes acercamientos analíticos y críticos en torno a los espacios tradicionales de socialización de los jóvenes, como la escuela, la familia, el trabajo, sin "perder" al sujeto juvenil. ${ }^{16}$

El desencuentro entre la producción de conocimiento de la vertiente que se ocupa de los "no-institucionales" y la que se ocupa de los "incorporados" es profundo y da como resultado, para una y para otra, análisis parciales en las que hay por un lado insuficiente tratamiento de los aspectos estructurales e institucionales no necesariamente antagónicos a las expresiones culturales juveniles y, por el otro, focalización en la institución en detrimento de la especificidad juvenil. De un lado sujetos sin estructura, del otro, estructura sin sujetos.

Un nuevo filón, que pudiera constituirse como puento de equilibrio entre estas perspectivas, lo constituyen los estudios que se ocupan del consumo cultural juvenil.

La relación con los bienes culturales como lugar de la negociación-tensión con los significados sociales. El consumo cultural como forma de identificacióndiferenciación social (García Canclini, 1993a; Bourdieu, 1988) que coloca al centro del debate la importancia que en términos de la dinâmica social tiene hoy en día la consolidación de una cultura-mundo que repercute en los modos de vida, los patrones socioculturales, el aprendizaje y fundamentalmente en la interacción social.

Estos estudios han mostrado al joven como un actor posicionado socioculturalmente y han abordado las interrelaciones entre los distintos ámbitos de pertenencia del joven - la familia, la escuela, el grupo de pares -, que se constituyen en comunidades inmediatas de significación (Orozco, 1991) y aquellos movimientos o "gramáticas de vida" en el

${ }^{16}$ Por ejemplo Reality bites, La sociedad de los poetas muertos, Breakfast club y Santana, americano yo?, esta última, conjunta la problemática de los migrantes latinoamericanos con la juvenil y cuestiona severamente el orden institucional. sentido habermasiano (Habermas, 1989), que hacen las veces de "comunidades imaginarias" a las cuales adscribirse. ${ }^{17}$

\section{Lo tematizable: segunda visitación}

"La caída de tabiques entre disciplinas" (Canclini, 1993) y la emergencia y paulatina consolidación de estudios llamados interdisciplinarios o "de frontera", han sido una constante en los ultimos años de investigación sobre juventud en América Latina.

Los contornos imprecisos del sujeto y sus prácticas han colocado al centro de los análisis la vida cotidiana no necesariamente como tema, sino como lugar metodológico desde el cual interrogar a la realidad.

Desde esta mirada, que se sitúa en los propios territorios de los jóvenes, los objetos-problema abordados han sido diversos, aqui se analizan centralmente cuatro que son los que a nuestro juicio dan sentido a la literatura especializada y conectan con la problemática que aqui nos ocupa: se trata del grupo y las diferentes maneras de entender y nombrar su constitución; el "otro construido" en relación con el proyecto identitario juvenil; la cultura política y la acción; y finalmente, la noción de futuro.

\section{El grupo}

La problematización en torno a "los modos de estar juntos" (Martín Barbero, 1995) de los jóvenes ha sido elaborada de diversas maneras.

La diferenciación más clara se relaciona con la direccionalidad del enfoque. Es decir, un tipo de estudios va de la constitución grupal a lo societal; otro tipo va de los ámbitos sociales al grupo.

En el caso del primer enfoque, la identidad grupal particular se convierte en el referente clave que permite "leer" la interacción de los sujetos con el mundo social. Hay por lo tanto un colectivo empírico al que

\footnotetext{
${ }^{17}$ Para un acercamiento a este tipo de identidades juveniles,
} véase José Manuel Valenzuela (1988) y Reguillo (1997). 
se observa y desde el cual se analizan las vinculaciones con la sociedad. A este tipo, por ejemplo, correspondeu las etnografías de bandas juveniles que centraron la atención durante la década de los ochenta.

Por razones del propio enfoque, para conceptualizar (pocas veces de manera explícita) la agregación juvenil, se ha recurrido a categorías como "identidades juveniles", "grupo de pares", "subculturas juveniles"; y las más de las veces, sobre todo durante la primera mitad de la década de los ochenta, se utilizó "banda" como "categoría" para nombrar el modo particular de estar juntos de los jóvenes populares urbanos. Esta mirada intragrupal, si bien ha aportado muy importantes elementos de comprensión, ha sido insuficiente para captar las vinculaciones entre lo local y lo global y las interacciones culturales.

Por otra parte, han ido cobrando fuerza los estudios que van de los âmbitos y de las prácticas sociales a la configuración de grupalidades juveniles. El rock, el uso de la radio y la televisión, la violencia, la política, el uso de la tecnología, se convierten aquí en el referente para rastrear relaciones, usos y decodificaciones y recodificaciones de los significados sociales de y para los jóvenes. No necesariamente debe existir entonces un colectivo empírico, se había de los "jóvenes de clase media", de los "jóvenes de los sectores populares" etc., que se constituyen en "sujetos empíricos" por la mediación de los instrumentos analíticos; se trata de "modos de estar juntos" a través de las prácticas que no se corresponden necesariamente con un territorio o un colectivo particular.

Esta vertiente ha buscado romper con los imperativos territoriales y las identidades esenciales y para ello ha construido categorías como la de "culturas (en plural) juveniles", "adscripción identitaria”, "imaginarios juveniles" (pese a lo pantanosa que resulta esta última). Es una mirada que trata de no perder al sujeto juvenil pero se busca entenderlo en sus múltiples "papeles" e interacciones sociales.

\section{El “otro"}

Un tema recorrente en los estudios sobre juventud, no por obsesión de los analistas sino porque apa- rece de manera explícitamente formulada por los jóvenes, es el de lo que aquí se denomina "el otro", para hacer referencia al "antagonista", o "alteridad radical”, que otorga más allá de las diferencias, por ejemplo, socioeconómicas y regionales, un sentimiento de pertenencia a un "nosotros". La identidad es centralmente una categoría de carácter relacional (identificación-diferenciación) y todos los grupos sociales tienden a instaurar su propia alteridad. La construcción simbólica "nosotros los jóvenes", ha instaurado diferentes alteridades, principalmente respecto al mundo adulto.

Diferentes estudios se han ocupado de construir corpus de representaciones en los que es posible analizar las separaciones, las fronteras, los muros que las culturas juveniles construyen para configurar sus mundos. Más allá de la dimensión antropoformizada de esas alteridades (policia, gobierno. maestros, escuela), algunos trabajos - que trascienden lo puramente descriptivo - han señalado que estas figuras representan para los jóvenes un orden social represor y por consiguiente injusto, se trata de los guardianes del orden; lo que aquí puede representar una obviedad, que no lo es tanto si se atiende a que buena parte de la literatura sobre juventud se ha quedado atrapada en el dato empírico, en la anecdota y que no separa la "militancia" en la lucha por los derechos humanos de los jóvenes de la tarea de producir conocimiento. ${ }^{18}$

${ }^{18}$ En diversas y numerosas reuniones donde se abordan temas relacionados con la juventud, muchos asistentes demandan que se hable un lenguaje "común", que "se renuncie a la teoría", que se hable de las cosas que "verdaderamente afectan a los jóvenes", en una especie de populismo que confunde espacios y fines. Ello ha obstaculizado no sólo en el caso de los jóvenes, sino también en el de las mujeres, los indígenas y algunas otras "minorías", la posibilidad de un debate riguroso en torno al pensamiento. Lo que no equivale a señalar que el trabajo de intervención sea fundamenal y que hay ocasiones que ameritan "salir" de la academia para entrar en el terreno de la política. Un trabajo reciente que concilia estas dos posiciones es el excelente estudio de Héctor Castillo, Juventud, cultura y política social, (en prensa). 


\section{Cultura política}

Algunos de los enfoques clásicos en torno a la conceptualización de la acción colectiva han centrado prioritariamente su mirada en aquellas maneras de participación formales, explícitas, orientadas y estables en el tiempo, ${ }^{19}$ con la consecuente teorización que parece reconocer sólo como cultura política aquellas representaciones y formas de acción formales y explícitas. Este tipo de intelección ha provocado que las grupalidades juveniles, efímeras, cambiantes, implícitas en sus formulaciones, sean leídas como carentes de un proyecto político y que se reduzca su relación en este ámbito, por ejemplo, a la participación electoral. ${ }^{20}$

Paulatinamente y en relación con la literatura sobre nuevos movimientos sociales y las reconceptualizaciones sobre lo político (Touraine, 1994; Melucci, 1989; Offe, 1990; Maffesolli, 1990; Sartori, 1992; Lechner, 1995), aparece en la literatura sobre juventud una revaloración de lo político, que deja de estar situado más allá del sujeto, constituyendo una esfera autónoma y especializada y adquiere corporeidad en las prácticas cotidianas de los actores, en los intersticios que los poderes no pueden vigilar (Reguillo, 1996).

La política no es un sistema rígido de normas para los jóvenes, es más bien una red variable de creencias, un bricolage de formas y estilos de vida, estrechamente vinculada a la cultura, entendida como "vehículo o medio por el que la relación entre los grupos es llevada a cabo" (Jameson, 1993).

Sin embargo, es importante reconocer que las articulaciones entre culturas juveniles y política están lejos de haber sido finamente trabajadas y que en términos generales esto se ha construido desde una relación de negatividad, es decir, desde la negación o

${ }^{19}$ Por ejemplo, el primer Touraine (1984).

${ }^{20}$ Por ejemplo, véase el estado de la cuestión en torno al tema político en los jóvenes, realizado por Ricardo Becerra Laguna, Participación política y ciudadana, en Pérez Islas y Maldonado (1996) desconocimiento de los constitutivos políticos en las representaciones y acciones juveniles. ${ }^{21}$

Lo quc el mapa aquí trazado intenta es revelar las fortalezas y debilidades en los estudios sobre juventud desde la perspectiva de los estudios culturales. Del conjunto de posibilidades de análisis, estos tres objetos-recortes se articulan a varias de las preguntas clave de los estudios culturales: la identidad como lugar de enunciación sociopolítica, las intersecciones entre prácticas y estructuras; los escenarios del conflicto y la negociación por la inclusión, vinculados tanto a los discursos como a las practicas y las coordenadas espacio-temporales como dimensiones constitutivas de lo social.

Resulta urgente hacer la crítica de los modos de conocimiento, del papel no inocente de la mirada que construye el conocimiento para elaborar una agenda que sin autocomplacencias permita trascender las visiones que han construido al joven como la pobre "víctima" de un orden injusto, como jinete del apocalipsis o como redentor.

La diferencia entre el discurso del sentido común respecto al discurso de las ciencias sociales es que el primero, para funcionar, requiere ser inconsciente (Ibáñez, 1994), aceptar el orden de las cosas como dato dado; mientras que el segundo supone la reflexividad mediante la crítica de los conceptos y las categorías.

\section{Los escenarios de fin de siglo}

La discontinuidad "autoriza" los compromisos efímeros, el cambio de banderas, y potencia la capaci-

${ }^{21}$ Creo firmemente que los zapatistas y el subcomandante Marcos han sabido captar (y aprovechar) con precisión este sentido polifónico de lo político en los jóvenes. Por ejemplo, los programas especiales en MTV Latino, la muy reciente "Canción del Sup", en la que a ritmo de rock, el Sup "rapea" las consignas zapatistas "para todos, todo"; que seguramente le costará severas críticas, tanto de las derechas como de las izquierdas, incapaces por distintos motivos - de entender la fuerza de la videopolitica y de los nuevos mecanismos de interpelación a los jóvenes. 
dad de respuesta en la medida en que se reduce el conflicto entre "habitar" una identidad u otra, entre defender una "causa" u otra. Mutantes de fin de siglo, los jóvenes tal vez no saben qué es lo que quieren pero saben muy bien qué es lo que no quieren. Es desde estos cambiantes sentidos por donde hay que pensar las culturas juveniles y sus sentidos sociales de la vida.

El que muchos de los jóvenes no opten por prácticas y formas de agrupación partidistas o institucionales y el hecho de que no parezcan ser portadores de proyectos políticos explícitos, desde una perspectiva tradicional, puede ocultar los nuevos sentidos de lo político que configuran redes de comunicación desde donde se procesa y se difunde el mundo social. Frente al "resplandor de lo publico", muchos de estos mutantes optan por la sombra, por el deslizamiento sigiloso, algunos para señalar la crisis, otros para hacer las paces con un sistema del que se sirven instrumentalmente.

$\mathrm{Al}$ iniciarse la década de los años noventa se consolidaron o se aceleraron algunas de las tendencias que venían anunciándose desde la década anterior, esto es: la mundialización de la cultura por via de las industrias culturales, los medios de comunicación y las supertecnologías de información (Internet es el ejemplo más acabado, aunque no el único); el triunfo del nuevo profetismo globalizador, el discurso neoliberal montado sobre el adelgazamiento del Estado y sobre la exaltación del individualismo; el empobrecimiento creciente de grandes sectores de la población; descrédito y deslegitimación de las instancias y dispositivos tradicionales de representación y participación (especialmente los partidos políticos y los sindicatos).

Estos elementos han significado para los jóvenes una afectación en: a) su percepción de la política, b) su percepción del espacio y c) su percepción del futuro

Situados en los márgenes de la sociedad - objetiva o simbólicamente -, los jóvenes, pese a las diferencias (de clase, de género, de emblemas aglutinadores) comparten varias características que pueden considerarse definitorias de las culturas juveniles en este fin de siglo:
1. Poseen una conciencia planetaria, globalizada, que puede considerarse como una vocación internacionalista. Nada de lo que pasa en el mundo les es ajeno, se mantienen conectados a través de complejas redes de interacción y consumo.

2. Priorizan los pequeños espacios de la vida cotidiana como trincheras para impulsar la transformación global.

3. Existe un respeto casi religioso por el individuo que se convierte en el centro de las prácticas. Puede decirse que la escala es individuo-mundo y que el grupo de pares no es ya un fin en sí mismo, sino una mediación que debe respetar la heterogeneidad.

4. Selección cuidadosa de las causas sociales en las que se involucran.

5. El barrio o el territorio han dejado de ser el epicentro del mundo.

Estas características representam un cambio frente a la década pasada.

En los ochenta, para los jóvenes de los sectores populares, el grupo de pares servía para cobijar, bajo un manto homogéneo, las diferencias individuales y ofrecer el efecto ilusorio de un nosotros compacto que se convertia en el punto de llegada y salida de las visiones del mundo; el barrio transformado por complicados mecanismos rituales de bautizo en territorio apropiado representaba el "tamaño del mundo"; la identidad colectiva hundía sus raíces en el territorio que servía simultáneamente como frontera que delimitaba lo interior-propio con lo exterior-ajeno.

Para los jóvenes de los sectores medios y altos, los ochenta fueron una década perdida. Herederos del desencanto político, del descrédito de las grandes banderas, muchos de ellos cómplices involuntarios de relatos paralizantes adoptaron a posteriori la denominación "generación X", que peligrosamente saltó del título de una novela del canadiense Douglas Coupland (Generation X, publicada en 1991) a una "categoría" que ha servido para definir el nihilismo, el consumismo, la depresión profunda y la renuncia al futuro de los jóvenes de los sectores acomodados 
del norte pero que penetró rápidamente las fronteras nacionales en América Latina y sirvió como un discurso post facto para justificar el tamaño del desinterés, la desarticulación y el desencanto de los jóvenes universitarios, yupies o empleados a tiempo completo como hijos de familia "porque qué flojera, o no hay trabajo".

En los noventa, las culturas juveniles, en su interacción con los otros, con la sociedad, son vistas de maneras también diversas. Para ciertas "lecturas", los jóvenes son desechables en tanto sujeto politico, motivo de "apañón" y de sospecha; botín electorero en tiempos de secas; espejo vergonzoso de la sociedad; objetos de reglamentos y planes; y, lamentablemente objetos - que no sujetos - de los discursos conmovedores de funcionarios y primeras damas en turno. Desde otras "lecturas", los jóvenes son vistos como personajes de novelas y películas, emblemas libertarios, potencia pura. Descalificación o exaltación.

Y mientras eso sucede, las culturas juveniles de la crisis, de la globalización y la tribalización, (re)inventan mecanismos para confortarse colectivamente y sobrevivir a la violencia cotidiana y generalizada, al desencanto profundo que les ha abierto un hoyo negro en la esperanza.

Éste es parte del paisaje social que se constituye simultáneamente en plataforma y motivo de análisis.

\section{Hacia una agenda de investigación}

Los jóvenes escapan a la definición cerrada, homogeneizadora, light que el discurso dominante impulsa a través de sus centros de irradiación y se resiste a la normatividad estatal. Más allá de la diversidad, lo que aquí importa destacar es que quizás la juventud sea, como lo ha señalado Feixa (1993), una metáfora del cambio social. Una llamada de atención, alerta roja, que nos obliga a repensar muchas de las certezas construidas.

Sistemáticamente en los estudios, en los planes, el "deber ser" ha monopolizado la comprensión, ignorando la capacidad de respuesta, las constantes "chapuzas" con la que los actores sociales (no sólo los jóvenes) se enfrentan al ordem establecido. $\mathrm{Su}$ nomadismo económico, territorial y cultural, porque no hay de otra, son formas, intentos de cerrarle el paso a la crisis, de luchar contra el estallamiento de certezas, domesticar la imprevisibilidad que dicen disfrutar.

Ni el Estado ni los partidos políticos han sido en lo general - capaces de generar matrices discursivas que puedan interpelar a los jóvenes.

Para ellos la construcción de lo político pasa por otros ejes: el deseo, la emotividad, la experiencia de un tiempo circular, el privilegio de los significantes por sobre los significados, las prácticas arraigadas en el ámbito local que se alimentan incesantemente de elementos de la cultura globalizada.

"Quieren amores posibles" (Muñoz, 1992), el debilitamiento del futuro deja lugar a la certidumbre del presente, de lo tangible. Y pese a la marginación, a la desesperanza y al miedo, apuestan por la vida.

Los jóvenes viven continuamente en la recomposición de prácticas y representaciones. Los esquemas explicativos procurados desde las ciencias sociales son insuficientes ya para dar cuenta de los acelerados cambios que se han operado, es urgente repensar muchas de las afirmaciones que se han hecho.

Los grandes medios de comunicación, con su vocación presentista, agotan los procesos sociales en el día a día; las autoridades, con su vocación correctiva, llegan cuando los jóvenes se vuelven "visibles" en función del problema que representan. El investigador, a su vez, trabaja a contracorriente. La mayor parte de las veces sus "objetos" no son necesariamente "noticias" en el sentido caliente del término, ni tematizables para ocupar los titulares de la prensa. En la década de los ochenta varios investigadores del país hablábamos de los jóvenes, el tema resultaba un tanto "exótico", llamativo en tanto se hacía alusión a un tipo de actor social, con una vestimenta, un lenguaje y unas propuestas organizativas poco ortodoxas. Pero más allá de esto, autoridades, medios de comunicación y desafortunadamente muchos investigadores, fueron incapaces de transcender las interesantes y efectivamente seductoras formas exteriores de estas identidades juveniles. Y lo que varios planteamos, en 
términos tanto culturales como de formas de socialidad, quedó eclipsado.

La generación que toma el relevo en los noventa vive y experimenta el mundo de maneras diferentes: han tomado la ciudad por asalto, pasaron del sedentarismo a un nomadismo cultural y territorial; están buscando nuevas formas de relación entre géneros y, si bien pueden parecer más violentos y desesperados, son más generosos con la noción de futuro.

¿Se puede hoy hablar de culturas juveniles?, ¿qué sabemos?, ¿qué debiéramos saber?, ¿qué perspectivas de estudio se abren, a partir de qué ejes?

Trazar una agenda de investigación por decreto no es ni factible ni recomendable. Así que la intención de esta última parte es apenas la de señalar algunos de los huecos en la investigación sobre juventud y apuntar algunos elementos de reconfiguración en los mundos juveniles.

Quizá la temática más ausente y extrañada sea la perspectiva de género en los estudios sobre juventud. Pese a las novedades que comportan las culturas juveniles, en lo que toca a las relaciones de género, éstas no han sido suficientemente abordadas. Si bien las y los jóvenes comparten universos simbólicos, lo hacen desde la diferencia cultural constituida por el género. La organicidad alcanzada por los colectivos juveniles de composición mayoritariamente masculina no es equivalente al caso de las jóvenes, que según muestran algunos de los estudios, tienden a insertarse en las grupalidades juveniles "masculinizándose". Pero hay insuficiencia de material empírico que permita hacer planteamientos finos en lo que toca a la diferencia de género entre los jóvenes.

El centralismo en las ciencias sociales que favorece la concentración de recursos para la investigación, de centros y de investigadores, entre otros factores, ha provocado que la especificidad regional de las culturas juveniles no haya sido suficientemente abordada. Tampoco se ha logrado avanzar sustancialmente en lo que toca a las dimensiones local-global y sus repercuciones en el ámbito de las culturas; cabe aquí preguntarse ¿cómo reformulan desde lo local los elementos de la cultura-mundo y cómo actualizan en la vida coti- diana las relaciones entre tradición y modernidad?

De un lado la victimización del joven y de otro lado su exaltación como agente de cambio polarizan, en términos generales, la investigación. El efecto que esto ha tenido es la de una diversidad fáctica sin problematización. Es decir, el "otro construido", tanto para los jóvenes como en relación con el discurso social que sobre ellos se elabora y circula, se asume como un dato que está ahí a la espera del observador. En tal sentido, hace falta investigación sobre los mapas cognitivos, sobre las experiencias mediatas e inmediatas de donde se nutren las representaciones colectivas que dan forma y contenido a las identidades-alteridades sociales. Especialmente en este momento, los poderes, particularmente la institucionalidad mediática, se disfrazan de ludismo y de espacio de conversación, haciendo aparecer "la diferencia" como un asunto retórico que oculta la desigualdad.

Esto apunta también a la necesidad urgente de investigaciones que, sin renunciar a la dimensión intragrupal, sean capaces de ver al joven más allá de los ámbitos restringidos de sus respectivos colectivos.

El balance realizado, si bien señala una tendencia creciente a los acercamientos interdisciplinarios, revela, de otro lado, una escasa problematización del sujeto juvenil desde las dimensiones psicosociales que no se reduzcan al establecimiento a priori de una serie de etapas y actitudes que caracterizan el periodo de la juventud. El problema es mucho más complejo y exigiría un trabajo más fino en los intefaces entre individuo, grupo y contexto sociocultural. En tal sentido, la perspectiva psicoanálitica ha sido una veta poco explorada en el campo de los estudios de la juventud.

Por último, y en el espíritu de fomentar la discusión, está la urgente necesidad de hacer estudios comparativos como una de las alternativas para propiciar el diálogo y un debate no virtual que pueda romper el aislamiento en la producción de conocimiento.

Es en esta dimensión donde cobra sentido el pensamiento de Ibáñez, "pensar juntos el pensamiento con el que pensamos". 
ROSSANA REGUILLO é licenciada e mestra em comunicação pelo ITESO - Instituto Tecnológico de Estudos Superiores do Ocidente e doutora em ciências sociais pela Universidade de Guadalajara. É professora do Departamento de Estudios de la Comunicación Social, na Universidad de Guadalajara e também do Departamento de Estudios Socioculturales. Atua como pesquisadora na área da cultura e da comunicação, especializada em estudos sobre juventude, no ITESCO. Conta com ampla produção divulgada tanto em publicações nacionais mexicanas como em publicações internacionais. E-mail : rossana@iteso.mx

\section{Referencias bibliográficas}

BOURDIEU, Pierre, WACQUANT, Loïc J. D., (1995). Respuestas; por una antropología reflexiva, México: Grijalbo.

BOURDIEU, Pierre, (1990). La juventud no es más que una palabra. Sociología y cultura, México: CNCA-Grijalbo (Colección Los Noventa). , (1988). La distinción. Madrid: Taurus.

CASTILLO, Hector, ZERMEÑO, Sergio, ZICCARDI, Alicia (1995). Juventud popular y banda en la Ciudad de Mexico. In : GARCÍA CANCLINI, Néstor (coord). Cultura y pospolítico; el debate sobre ma modernidad en América Latina. México: CNCA. p. 273- 294.

DUQUE, J. R., MUÑOZ, B., (1995). La ley de la calle : testimonios de jóvenes protagonistas de la violencia en Caracas. Caracas: Fundarte.

FEIXA, Carles, (1993). La juventud como metafora; sobre las culturas juveniles, España: Generalitat de Catalunya.

GARCÍA CANCLINI, Néstor, (coord.), (1993a). El consumo cultural en México. México: CNCA (Colección Pensar la Cultura). , (1993b). Introducción: antropología y estudios culturales. Alteridades. México: UAM-I. n ${ }^{\circ} 5$.

(1995). Consumidores y ciudadanos; conflictos multiculturales de la globalización. México: Grijalbo.

GIDDENS, Anthony, (1995). Beyond left and right. Cambridge: Polity. (1986). The constitution of society. Los Angeles: University of California Press.
GÓMEZ JARA, Francisco et al., (1987). Las bandas en tiempo de crisis. México: Nueva Sociologia.

HABERMAS, Jürgen, (1989). Teoría de la acción comunicativa: complementos y estudios previos. Madrid: Cátedra.

IBÁÑEZ, Jesús, (1994). El regreso del sujeto; la investigación social de segundo orden. Madrid: Siglo XXI.

IZQUIERDO, Rafael, (1996). Juventud y empleo: entre la escuela y el trabajo. In: CORDERA, Rafael Campos, BECERRA, Ricardo (coords). México joven; políticas y propuestas para la discusión. México: UNAM. p. 117-146.

JAMESON, Frederic, (1993). Conflictos interdisciplinarios en la investigación sobre cultura. Alteridades, México: UAM-I, $\mathrm{n}^{\circ} 5$.

LECHNER, Norbert, (1990). Los patios interiores de la democracia; subjetividad y política. México: FCE , (1995). Por qué la política ya no es lo que era. $\mathrm{Ne}$ xos, México, p. 23-32.

LEVI, Giovanni, SCHMITT, Jean Claude, (1995). História dos jovens. São Paulo: Companhia das Letras.

LUENGO, Enrique, (1996). Valores y religión en los jóvenes. In: PÉREZ ISLAS, José Antonio, MALDONADO, Elsa Patrícia. Jóvenes : una evalución del conocimiento; la investigación sobre juventud en México, 1986-1996. México: Causa Joven, p. $165-189$.

MAFFESOLI, Michel, (1993). El conocimiento ordinario; compendio de sociología, México: FCE. , (1990). El tiempo de las tribus. Barcelona: Iearia.

MARSHALL, T. H., (1965). Class, citizenship and social development. Nueva York: Anchor Books.

MARTÍN BARBERO, Jesús (1995). Pre-textos; conversaciones sobre la comunicación y sus textos. Cali: Centro Editorial Universidad del Valle.

, (1993). La comunicación en las transformaciones del campo cultural. Alteridades, México: UAM-I, n 5 .

MELUCCI, A., (1989). Nomads of present; social movements and individual needs in contemporary society. Philadelphia: Temple University Press. , (s/d). En busca de la acción (mimeo). 
MONSIVÁIS, Carlos, (1988). Escenas de pudor y liviandad. México: Grijalbo.

MUNÕZ, Sonia, (1992). Mundos de vida y modos de ver. In: BARBERO, Jesús Martín, MUÑOZ, Sonia (coords). Television y melodrama; generos y lecturas de la telenovela en Colombia. Bogotá: Tercer Mund Editores.

OFFE, Claus, (1990). Contradicciones del Estado de bienestar. México: CNCA/Alianza Editorial (Colección Los Noventa).

OROZCO, Guillermo, (1991). Recepción televisiva. México: Proicom, Universidad Iberoamericana.

PIKE, K. L., (1954). Language in relation to a unified theory of the structure of human behavior. Glendale: Summer Institute of Linguistic.

REGUILLO, Rossana, (1991). En la calle outra vez las bandas: identidad urbana y usos de la comunicación. Guadalajara: ITESO

, (1993). Notas críticas sobre los movimientos sociales: una perspectiva gramsciana. Iztapalapa, México: UAM-I, $\mathrm{n}^{\circ} 30$, jul.-dic.

, (1996). La construción simbólica de la ciudad; sociedad, desastre y comunicación, Guadalajara: Universidad Iberoamericana/ITESO,

, (1997). Culturas juveniles, producir la identidad: un mapa de interaccionaes. Jovenes. Revista de Estudios sobre Ju- ventud. México: Causa Joven/Centro de Investigación y Estudios sobre Juventud., año 2, n 5 , p. 12-31, jul.-dic., quarta época.

ROSALDO, Renato, (1990). Social analysis in history and anthropology. In: KAYE, Harvey J, MCLELLAND, Keith (eds.), E. P. Thompson: critical perspectives (mimeo).

ROUX, Gustavo I., (1994). Ciudad y violencia en América Latina. Quito: Gestión Urbana, v. 2.

SALAZAR, Alonso J., (1998). La cola del lagarto; drogas y narcotráfico em la sociedade colombiana.

SARTORI, Giovanni, (1992). Elementos de teoría política. Madrid: Alianza Editorial.

TOURAINE, Alain, (1994). Crítica a la modernidad. México: FCE. (1984). El regreso del actor. Buenos Aires: Eudeba.

URTEAGA-POZO, Maritza, (1993). Banda de subjetividades. In : AGUILLAR, De Garay, Simpósio por el rock. México: UAM Azcapotzalco. p. 50-65.

VALENZUELA, José Manuel, (1988). A la Brava ése! Tijuana: El Colégio de la Frontera Norte.

WALLERSTEIN, Immanuel (coord.), (1996). Abrir las ciencias sociales. Comisión Gulbenkian para la restructuración de las ciencias sociales México: Siglo XXI/UNAM.

Recebido em janeiro de 2003

Aprovado em março de 2003 